Editorial:

\title{
How can be Avoided Plagiarism in Original Research Articles?
}

Ankarali $H$

International Journal of Human and Health Sciences Vol. 02 No. 02 April'18 Page : 47-48

Plagiarism can be defined as the writing of information, ideas, thoughts, opinions and/or methods belonging to others without reference to those persons. In this case, the person reading the article thinks these information belongs to the person who wrote it. In addition, taking the main idea of the subject and rewriting it with word changes is regarded also as plagiarism.In brief, it would not be wrong to use the concept of "theft" in scientific writings for plagiarism.In this case plagiarism emerges as a moral problem.

There are two main reasons for plagiarism also, as in all other ethical violations. These are 1) Lack of experience and knowledge 2) Intentional plagiarism. A significant number of authors violate ethics rules due to lack of knowledge on plagiarism and/or other publication ethics, and/or inexperience in scientific researches. One another reason for plagiarism is the necessity of authors to prepare a scientific article other than their native language. One of the most important reason for the spread of plagiarism is that it is easy to access references on the internet and copy-paste is possible. It does necessary that researchers should be given trainings in order to reduce ethical violations on writings.

Plagiarism done especially in original research articles which is one of scientific research articles. The information obtained from thesis or individual researches should be prepared as the original research article type. Plagiarism lowers the quality and authenticity of scientific writings, and adapts the person to readiness. Several programs, such as iThenticate and Turnitin, are used for detection of plagiarism.

In the world of science, access to information is getting easier every day. For this reason, in order to minimize the risk of plagiarism, all the references, which are used, should be given on end of the all related paragraph or sentences and in the list of references together with all their information.If the quotation is to be used without any modification, the relevant statements must be given in quotation marks.

In this editorial, I would like to make a suggestion for the publishers such as scientific journals which publish especially scientific articles in order to prevent or minimize plagiarism. As I mentioned above, plagiarism is mostly confronted with when writing original research (thesis or individual research).Because the format of this type of articles is prepared according to the rule called IMRAD. Abbreviation of IMRAD; I: Introduction, M: Materials and Methods, R: Results, A: And, D: Discussion. Plagiarism is most commonly done in the Introduction and Discussion sections. Because literature knowledge is used in these two sections. In general, Material and Methods section has a small number of references are found and they mentioned in related part (methods developed by others).

In the preparation of Original Articles, we can say that writing a long introduction and discussion sections leads to unnecessary duplication of information. Instead of Introduction section, only the aims and originality of the article should be given. Instead of long discussions in the Discussion section, only Conclusions, Limitations and Recommendations should be given. All related references should be given in the References section and also the page and paragraph numbers of the information in this references that will be relevant to the study should be added. For example, you used as a reference $\boldsymbol{A}$ which is a review article and page numbers $85-93$ in print when writing your article.If this reference on page 86 , the second paragraph, on page 88 , the first paragraph and on page 91, the third paragraph is contained information about your research, in the References section, which is the last part of

Correspondence to: Prof. Handan Ankarali, Department of Biostatistics, Istanbul Medeniyet University, Istanbul, Turkey. Associate Editor, IJHHS E-mail:Handan.ankarali@medeniyet.edu.tr 
the article, the relevant reference can be given in a detailed format in the following way.

Sample Reference Format is,

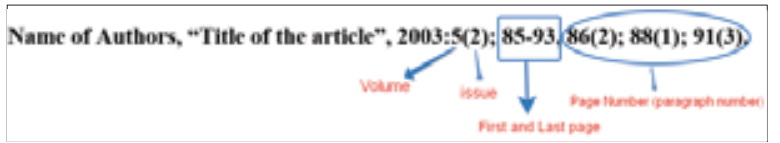

For this format, abbreviations AO: M: R: CLR or any other abbreviation may be suggested.A: Aims, O: Originality: M: Materials and Methods, R: Results, C: Conclusions, L: Limitations, R: Recommendation.Thus, the information to be written by recalling from other references is reduced to the minimum.Because the article prepared will now only contain information and findings specific to that study.If readers want to get more detailed information, they will be able to easily access the related pages and paragraphs defined in the references.In addition, a format should be created for all types of references that can be utilized and the internet access address must be shown in references list.

This proposal can be evaluated and discussed by various publisher or journal editors.Our goal is to reduce plagiarism to the least, and to ensure that the originality, recommendations and limitations of scientific articles are at the forefront.

With the wish of a science world without plagiarism... 